\title{
Control Oriented Modeling of the Gas Exchange Process in Variable Cam Timing Engines
}

\author{
Per Öberg and Lars Eriksson \\ Vehicular Systems, Dept of EE \\ Linköpings Universitet \\ S-581 83 Linköping \\ SWEDEN
}

Copyright (c) 2006 Society of Automotive Engineers, Inc.

\begin{abstract}
Variable cam timing engines pose new questions for engine control system designers. The cam timing directly influences cylinder air charge and residual mass fraction. Three models that predict residual mass fraction are investigated for a turbocharged dual independent Variable Cam Timing (VCT) engine. The three models (Fox et. al. 1993, Ponti et. al. 2002, and Mladek et.al. 2000) that all have real time capabilities are evaluated and validated against data from a crank angle based reference model. None of these models have previously been validated to cover this engine type.

It is shown that all three models can be extended to dual independent VCT engines and that they also give a good description of the residual gas fraction. However, it is shown that the two most advanced models, based on a thermodynamic energy balance, are very sensitive to the model inputs and proper care must therefore be taken when these models are used.
\end{abstract}

\section{INTRODUCTION}

Air charge is important for engine fuel and torque control while residual mass fraction is a crucial factor that limits stable engine operation since it influences the combustion variability. Therefore it is essential for the control system to know the air charge and residual mass fraction, hence models that are accurate enough are required. When cam timing is used to its full extent existing control oriented models for residual mass fraction and cylinder air charge have the shortcoming of not covering the entire engine operating region.

The three models (Fox etal. 1993 [1], Ponti et al 2002 [2] and Mladek et al. 2000 [3]) that are investigated in this paper all predict residual mass fraction. The model in [3] also predicts air charge. When it comes to applicability the model in [1] is stated to be applicable for low to medium engine speeds. In [3] no operating points where the Variable Valve Timing (VVT) system has been active are used in the estimating procedure. In [2] both VVT and External EGR is used in the model but the model has not been validated. None of these three models have previously been validated to for a dual independent variable cam timing engine.

There is thus a need to investigate if the models can describe the residual gas fraction for dual independent VCT engines and to validate them over the engines' full operating range.

There are other publications that propose control and or estimation algorithms for different types of VVT systems, but the focus has been on the air charge and not on the residual gas fraction. In [4] and [5] air charge for dual equal and intake only VCT systems, that have moderate valve overlap, is studied. In [6] the focus is on fuel injection for the same type of engines as mentioned earlier.

The models

Model A, Fox et al. 1993 [1], is a simple generalized flow restriction model created with physical insight. Two model parameters are tuned to measurements or simulations of residual mass fractions.

Model B, from Ponti et al 2002 [2], and Model C, from Mladek et al. 2000 [3], are both based on thermodynamic relations, e.g. an energy balance at IVC. They both require measurement of cylinder pressure.

The reference model that is used is a crank angle based multi-zone model. A reference model is necessitated by the fact that it is very difficult to directly measure the actual residual mass fraction in a production engine.

A straightforward validation of the residual mass fraction from the reference model is impossible. Therefore the reference model is tuned and validated against measured cylinder and manifold pressures, temperatures and mass flows through repeated simulations. Experimental data for the validation is obtained from a four cylinder, turbocharged engine with dual-independent continuously variable cam timing. 


\section{EXPERIMENTAL SETUP}

Measurements have been obtained for 2000 and $4000 \mathrm{rpm}$ using six different air masses at $7 \times 7$ different cam positions. The span in air mass and cam position is chosen to cover as much of the engine operating region as possible. Altogether 427 of the 588 possible operating points are used because the engine does not produce work for all combinations.

Intake and exhaust manifold pressures have been measured using standard pressure sensors. Temperatures in exhaust and intake manifolds are measured using standard thermo elements and mass flow into the engine have been measured using the built in hot-film air mass flow meter. The cylinder pressures has been measured with a resolution of one crank angle degree. The number of cycles for each measurement is more than 300 .

\section{REFERENCE MODEL}

As reference model a crank angle based zero-dimensional multi-zone model has been used. The model is tailored to track the flows of fresh and burned gases during the gas exchange period. It is built upon the method presented in [7] that solves the in-cylinder differential equations.

In the reference model the engine cycle is divided into three phases, gas exchange, compression and combustion. During the gas exchange and the combustion phase two zones are used. During the compression phase however only one zone is used.

The two zones in the gas exchange phase are used too separate the residual gas and the fresh charge. When entering the compression phase the gases are instantaneously mixed into one zone. In the combustion phase one zone is used to track the unburned gases and the other is used to track the burned gas. Combustion in modeled as a mass transfer from the unburned to the burned zone.

Flow into and out of the cylinder is modeled by standard flow restrictions with constant discharge coefficients and the valves open areas are modeled by the geometries and valve lifts from the actual engine. There are no dynamics in the exhaust or intake manifold and heating/cooling by the exhaust/intake ports is not considered. The fuel that enters the model is regarded as well mixed and no consideration is taken to charge cooling by evaporation.

In cylinder heat transfer is modeled using Woschni [8] with constant wall temperature. For burn ratio the standard Vibe [9] function has been used.

Thermochemical data for the model is obtained by using polynomials fitted to calculations using the chemical equilibrium program package, CHEPP, presented in [10]. For $c_{p}$ and $c_{v}$ dissociation is considered but the specific gas constant, $R$, is constant. The fuel used is isooctane.

\section{Model parameters}

Most of the parameters in the reference model is either kept constant for all operating points or taken from measurements in the specific point. The only parameters that are free are the parameters in the Vibe combustion function.

A complete list of parameters and constants used in the reference model can be found in Table 1 in Appendix B.

Fitting of Vibe parameters. The reference model is fitted to measurements using a least square optimization scheme. As residual the following measure is used

$$
r e s=\sqrt{\left(\frac{m_{a i r, s}-m_{a i r, m}}{m_{a i r, m}}\right)^{2}+\sum_{i}\left(\frac{p_{s, i}-p_{m, i}-p_{o}}{10^{6}[\mathrm{~Pa}]}\right)^{2}}
$$

where $m_{a i r, s}$ and $m_{a i r, m}$ represents measured and simulated cylinder air mass per combustion. $p_{s, i}$ represents samples of simulated cylinder pressure and $p_{m, i}$ represents measured. $p_{o}$ is the cylinder pressure offset that minimizes the residual in each optimization step.

The parameters that are sough for are $\theta_{s}, \theta_{e}$ and $m$ in the Vibe function. An initial estimate of these parameters is calculated using the techniques presented in [11].

It has to be stressed that all other parameters in the reference model are left as constants or calculated from measurements during the least square fitting. For example, there is no fitting of heat transfer coefficients or temperatures to make the residuals smaller.

Signal conditioning The data from the measurements is resampled and the crank angle offset from TDC estimated before the parameter fit. The crank angle offset is obtained using the same procedure as when fitting the Vibe parameters above. Here thermochemical data for pure air is used instead of an air/fuel mixture and the Vibe parameters are kept constant while the crank angle offset is free.

\section{Accuracy of reference model}

The reference model is used to obtain data for the other methods since they are used exclusively on simulated data. Even so it is interesting to see how well the reference model describes a real engine. The results from comparisons with measured data are found in Appendix B. The global agreement is good considered that most of the parameters are constants or measured values that are constant within the estimation procedure. 


\section{THE EVALUATED MODELS}

\section{Model A}

Model A, Fox et al. [1], defines an overlap factor $(O F)$ that measures the amount of overlap between intake and exhaust valves. The overlap factor is then used as effective area in a generalized flow restriction model. The model has two parameters that need tuning. These parameters are tuned to residual mass fractions from measurements or simulations. The following quantities are needed when using the model

$$
\begin{aligned}
& \text { - Intake manifold pressure, } p_{i m} \\
& \text { - Exhaust manifold pressure, } p_{e m} \\
& \text { - Engine speed, } N \\
& \text { - Fuel air ratio, } \Phi \\
& \text { - Valve positions (which gives } O F \text { ) }
\end{aligned}
$$

Computational scheme The key element in Model A is the overlap factor which is defined as

$$
O F=\frac{D_{i} A_{i}+D_{e} A_{e}}{V_{d}}
$$

where $D_{i}$ and $D_{e}$ are the inner seat diameters of the intake and exhaust valves and $V_{d}$ is the displacement volume of the engine. $A_{i}$ and $A_{e}$ are the areas under the valvelift/crank-angle curves and are defined as

$$
A_{i}=\int_{I V O}^{L_{i}=L_{e}} L_{i} d \theta \text { and } A_{e}=\int_{L_{i}=L_{e}}^{E V C} L_{e} d \theta
$$

where $L_{i}$ and $L_{e}$ are the intake and exhaust valve lifts respectively. In this implementation $L_{i}$ and $L_{e}$ have been calculated using the assumption that they are proportional to the valves effective flow areas, i.e. $L_{i} \propto \frac{A_{e f f, i}}{D_{i}}$ and $L_{i} \propto \frac{A_{e f f, e}}{D_{e}}$. Since the valve lifts in the calculation of $A_{i}$ and $A_{e}$ are small this has little effect on the calculation of $O F$.

Finally the residual mass fraction can be calculated using the following expression

$$
\begin{aligned}
x_{R G} & =C_{1 x_{R G}} \cdot\left(\frac{p_{e m}}{p_{i m}}\right)^{\left(\frac{\gamma+1}{2 \gamma}\right)} \cdot\left(\frac{O F}{N}\right) \cdot \sqrt{p_{e m}-p_{i m}} \\
& +C_{2 x_{R G}} \cdot \frac{1}{\lambda \cdot r_{c}}\left(\frac{p_{i m}}{p_{e m}}\right)^{\frac{1}{\gamma}}
\end{aligned}
$$

where $C_{1 x_{R G}}$ and $C_{1 x_{R G}}$ are constants that have to be fitted to data from measurements or simulations. These constants have been tuned to data from the reference model and the results are shown in Figure 14 in Appendix C.

\section{Model B}

Model B is presented in [2] and is a simplification of Model C. The model was originally used for an engine with ex- ternal EGR and VVT. External EGR is omitted in this implementation since the engine used lacks external EGR capabilities. The key element in the method is an energy balance at IVC. Using this energy balance the residual mass can be calculated from the following equation

$$
\begin{aligned}
& \left(m_{F C} c_{v, F C}+m_{R G} c_{v, R G}\right) T_{I V C}= \\
= & m_{F C} c_{v, F C} T_{F C}+m_{R G} c_{v, R G} T_{R G}
\end{aligned}
$$

as long as $T_{I V C}, T_{F C}$ and $T_{R G}$ are known. Estimates of $T_{F C}$ and $T_{I V C}$ are calculated using measurements of the following quantities

- In cylinder pressure, $p_{c y l}$

- Intake manifold temperature, $T_{i m}$

- Air mass entering the cylinder, $m_{F C}$

- Engine speed, $N$

A good estimate of $T_{R G}$, however, is trickier and is therefore obtained using a correlation between $T_{R G}$ and $m_{T o t}$. $N$.

Algorithm The first step of the algorithm is to adjust the cylinder pressure to the correct level. The reason for this is that the pressure measurements system used have a slowly varying offset. This adjustment is done by considering the cylinder pressure during the compression phase as a polythropic process for which $p \cdot V^{\gamma}$ is constant. A special variant of this technique is used in [2] but since the model is used on simulated data no compensation is needed and therefore the description of this technique is left out.

The temperatature at IVC is calculated using cylinder pressure at IVC, measured air charge, $m_{F C}$, an estimate of the residual mass fraction, $x_{R G}$, and the following equations

$$
\begin{aligned}
m_{T o t} & =\frac{m_{F C}}{1-x_{R G}} \\
R_{I V C} & =R_{R G} x_{R G}+R_{F C}\left(1-x_{R G}\right) \\
T_{I V C} & =\frac{p_{I V C} V_{I V C}}{R_{I V C} m_{T o t}}
\end{aligned}
$$

In the next step the temperature of the fresh charge has to be estimated. In [3] the estimation of the fresh charge temperature for this model is discussed in detail. It is stated that the heat flux from the walls to the fresh charge is partly compensated for the fuel evaporating process. Therefore, the fresh charge temperature can be estimated using a polythropic compression from manifold conditions to in-cylinder conditions.

$$
T_{F C}=T_{i m} \cdot\left(\frac{p_{i m}}{p_{I V C}}\right)^{\frac{1-\gamma_{F C}}{\gamma_{F C}}}
$$

When it comes to the temperature for the residual gas other measures have to be taken. In [3] a correlation between mass flow and burned gas temperature at IVC is used. The model is

$$
T_{R G}=-\left(C_{1 T_{R G}} \cdot\left(m_{T o t} \cdot N\right)\right)^{C_{2 T_{R G}}}+C_{3 T_{R G}}
$$




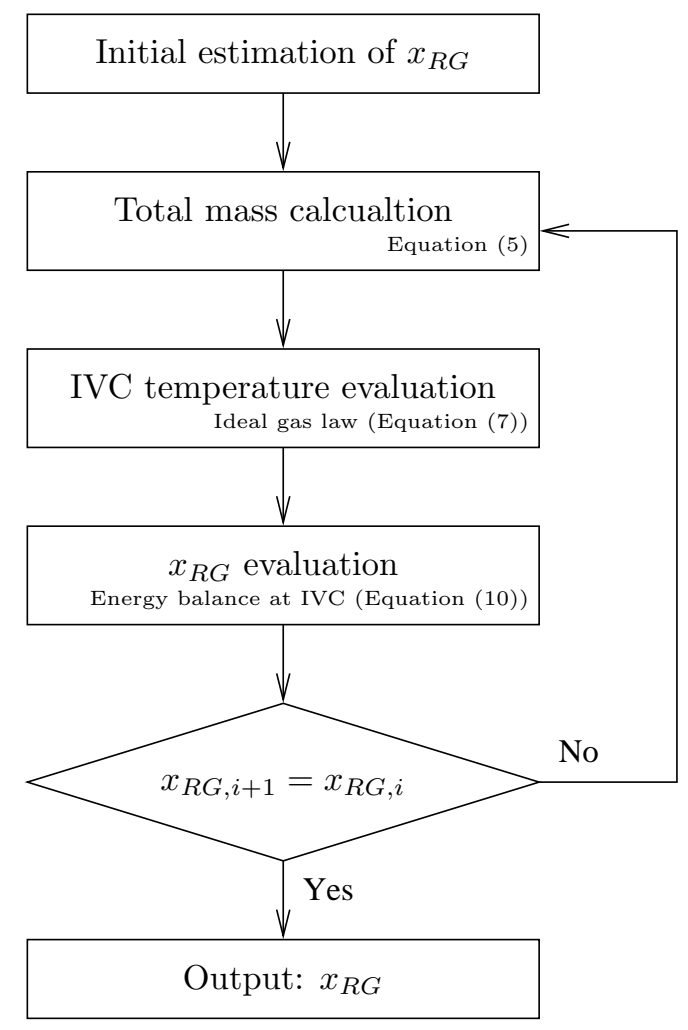

Figure 1: Flowchart of Model B. Using an initial estimate of $x_{R G}$ a new value can be obtained using Equation (5),(7) and(10). Repeating this in an iterative manner a final value of $x_{R G}$ is obtained.

where the three constants $C_{1 T_{R G}}, C_{2 T_{R G}}$ and $C_{3 T_{R G}}$ are estimated using simulations. These parameters have been tuned to data from the reference model and results from the tuning are shown in Figure 15 in Appendix C.

Finally the new residual mass fraction can be estimated using the energy balance at IVC in Equation (4)

$$
x_{R G}=\frac{c_{v, F C}\left(T_{I V C}-T_{F C}\right)}{c_{v, R G}\left(T_{R G}-T_{I V C}\right)+c_{v, F C}\left(T_{I V C}-T_{F C}\right)}
$$

where the specific heat values at constant volume $c_{v, F C}$, $c_{v, R G}$ and $c_{v, I V C}$ are the same as in the reference model.

Given an initial estimate of $x_{R G}$ and using Equation (5)(10) a new $x_{R G}$ can thus be calculated. The problem is solved by fixpoint iteration and gives the final estimate of $x_{R G}$. The iterative process is summarized by the flowchart in Figure 1.

Unfortunately the original fixpoint iteration does not always converge. In [2] this has been addressed and a stabilizing scheme has been suggested. Instead of updating the residual estimate with the new value in each iteration the following weighting is performed

$$
x_{R G, \text { used }}=x_{R G, \text { old }}+\frac{x_{R G, \text { new }}-x_{R G, \text { old }}}{2}
$$

\section{Model C}

Model C is presented in [3]. The key elements are energy balance equations at two different points during the engine cycle. Using these equations both cylinder air charge and residual mass fraction can be calculated. In addition to well known thermodynamic relations two approximations are used to get estimates of the residual mass temperature at IVC and the relative heat loss to cylinder walls at $x_{b}=$ $50 \%$.

The model requires on-line measurements of

- In cylinder pressure, $p_{c y l}$

- Intake manifold temperature, $T_{i m}$

- Engine speed, $N$

Original Algorithm Model C uses much of the same principles as Model B. As mentioned before Model B is in fact a simplification of Model $\mathrm{C}$. The difference is that the fresh charge, $m_{F C}$, is known in Model B but is estimated in Model C. Equation (6)-(10) is thus the same for Model C as for Model B.

One extra equation is needed to be able to estimate the total mass in the cylinder, $m_{T o t}$, that was considered as known in Model B. Energy balance at $x_{b}=50 \%$ is therefore introduced. The position for $x_{b}=50 \%$ is estimated using a burn rate analysis as follows:

1. The end of combustion is found by using the following relation $c a_{B E}=\operatorname{argmax}\left(p \cdot V^{1.15}\right)+10$.

2. Weighting values are calculated so that the final $x_{b}$ will be $0 \%$ at start of combustion and $100 \%$ at end of combustion. The weighting values are

$$
\begin{aligned}
& \dot{p}_{C S}=p_{C S}\left(\frac{V_{C S}}{V_{T D C}}\right)^{\gamma_{c o m p}} \\
& \dot{p}_{C E}=p_{C E}\left(\frac{V_{C E}}{V_{T D C}}\right)^{\gamma_{e x p}}
\end{aligned}
$$

where $\gamma_{c o m p}$ and $\gamma_{e x p}$ are the polythropic indices for compression and expansion phases respectively. Their values are set to 1.32 and 1.27 , as suggested in [3].

3. $x_{b}$ is then calculated as

$$
x_{b}=\frac{p\left(\frac{V}{V_{T D C}}\right)^{\gamma(c a)}-\dot{p}_{C S}}{\dot{p}_{C E}-\dot{p}_{C S}}
$$

where $\gamma(c a)$ is equal to $\gamma_{c o m p}$ for $c a \leq c a_{T D C}$ and $\gamma_{e x p}$ for $c a>c a_{T D C}$.

4. Finally the crank angle for $x_{b}=50 \%$ is obtained from the estimated $x_{b}$. 
The energy balance equation at $x_{b}=50 \%$ is formulated as follows

$$
\begin{aligned}
\bar{T}_{50} & =\frac{\int_{c a_{I V C}}^{c a_{50}} p d V}{m_{T o t} c_{v 50}}+\frac{\bar{T}_{I V C} c_{v I V C}}{c_{v 50}} \\
& +\frac{\left(1-q_{\text {Cooling }}\right) \cdot 0.5 \cdot x_{c} \cdot\left(1-x_{R G}\right) \cdot \frac{1}{1+\lambda \cdot A F_{s}} \cdot q_{H V}}{c_{v 50}}
\end{aligned}
$$

where $\bar{T}_{50}$ is the mean gas temperature at $x_{b}=50 \%$, $q_{\text {Cooling }}$ is a factor describing the heat loss to the cylinder wall, $x_{c}$ is a compensating factor for incomplete combustion, $A F_{s}$ is the stoichiometric air fuel ratio, $\lambda$ is the normalized air fuel ratio and $q_{H V}$ is the higher heating value of the fuel.

Since $q_{\text {Cooling }}$ is unknown it has to be estimated and this is done using the following correlation

$$
q_{\text {Cooling }}=C_{1 q_{\text {cool }}} \cdot \bar{T}_{50}-C_{2 q_{\text {cool }}}
$$

where $C_{1 q_{\text {cool }}}$ and $C_{2 q_{\text {cool }}}$ are constants that have to be tuned to data from simulations. Values obtained for the reference model can be found in Figure 16 in Appendix C.

Algorithm modification Using the $c_{v, 50}$ calculated from the gas composition, i.e. calculated in the same way as for the reference model, will not give accurate results when using the energy balance in Equation (13) above. This is because

$$
\Delta U=\int_{T_{1}}^{T_{2}} c_{v}(T) d T=m c_{v 2} T_{2}-m c_{v 1} T_{1}
$$

only holds if $c_{v}(T)$ is constant. Since $c_{v}(T) \approx c_{v 1}+$ $\frac{c_{v 2}-c_{v 1}}{T_{2}-T_{1}}\left(T-T_{1}\right)$ for the typical temperature interval Equation (13) has been modified in this implementation. Another issue is that $\int_{c a_{I V C}}^{c a_{50}} p d V$ is negative up till TDC since the volume is decreasing. Since work is added to the gas during this period a sign shift is necessary for the equation to be correct. The final expression is therefore

$$
\begin{aligned}
\bar{T}_{50} & =T_{I V C}-\frac{\int_{c a_{I V C}}^{c a_{50}} p d V}{m_{T o t}\left(c_{v, I V C}+c_{v, 50}\right) / 2} \\
& +\frac{\left(1-q_{\text {Cooling }}\right) \cdot 0.5 \cdot x_{c} \cdot\left(1-x_{R G}\right) \cdot \frac{1}{1+\lambda \cdot A F_{s}} \cdot q_{H V}}{\left(c_{v, I V C}+c_{v, 50}\right) / 2}
\end{aligned}
$$

Finally, when $\bar{T}_{50}$ is known a new total mass estimate can be performed. Since no dissociation is considered in the reference model the gas constant for the composition is straight forward to calculate and is equal to $R_{I V C}$. Hence the total mass in the cylinder can be calculated using the relations

$$
\begin{aligned}
R_{50} & =R_{I V C} \\
m_{T o t} & =\frac{p_{50} V_{50}}{R_{50} T_{50}}
\end{aligned}
$$

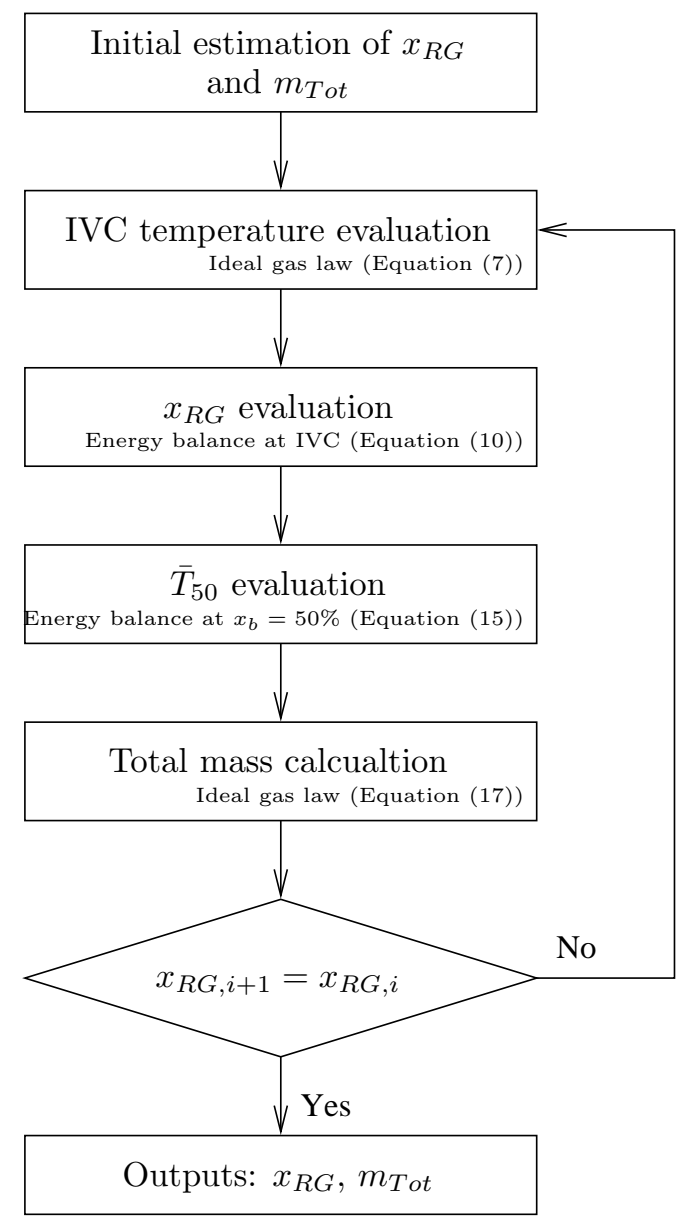

Figure 2: Flowchart of Model C. Using an initial estimate of $x_{R G}$ and $m_{T o t}$, new values can be obtained using Equation (7), (10), (15) and (17). Repeating this in an iterative manner final values of $x_{R G}$ and $m_{T o t}$ is obtained.

Complete algorithm The residual mass fraction, $x_{R G}$, can be calculated using an initial estimate of $x_{R G}$ and the total mass in the cylinder, $m_{T o t}$, much in the same way as in Model B. A flowchart of the process is shown in Figure 2. 


\section{RESULTS}

\section{Model A}

Given the simplicity of Model A it performs rather well. Looking at Figures 3(a) and 3(b) it can be noted that the relative error is well under $70 \%$. Studying the figures closer however reveals that there are effects that are not captured by the model since the errors have systematic content.

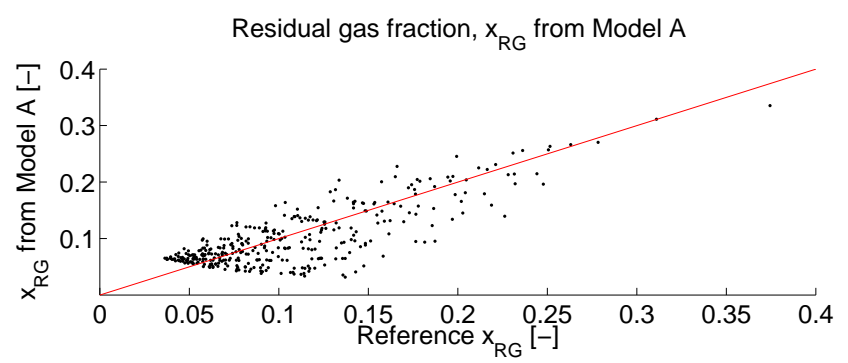

(a)

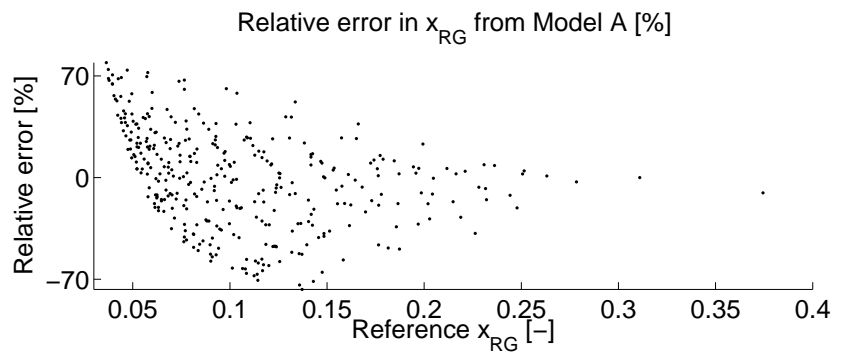

(b)

Figure 3: At first sight the modeled residual fraction gives a rather good fit to the reference. Studying the figure closer however reveals that there are effects that are not captured by the model (a). The relative error for Model A is well under $70 \%$ (b). Given the simplicity of the model this has to be considered as a good result.

The reasoning behind this conclusion is that if the model captured all physical effects the error should look more like noise and have no systematic content. Another issue with the model is that it uses the square root of the difference in manifold pressures in Equation (3). Since this value is not always positive for a turbocharged engine problems arise. It is not obvious how to include this into the model and therefore these points have been left out from the estimation.

\section{Model B}

Studying the residual mass fractions from Model B in Figure 4 it is evident that the model have some difficulties for small residual fractions. A comparison with the relative error for Model A in Figure 3(b) shows that the error is even larger than for Model A.

Since the greater part of the error is an offset and a scale error it is interesting to try and find the cause of the problems. Analyzing the method a number of observations can be made.
Residual gas fraction, $\mathrm{x}_{\mathrm{RG}}$ from Model $\mathrm{B}$

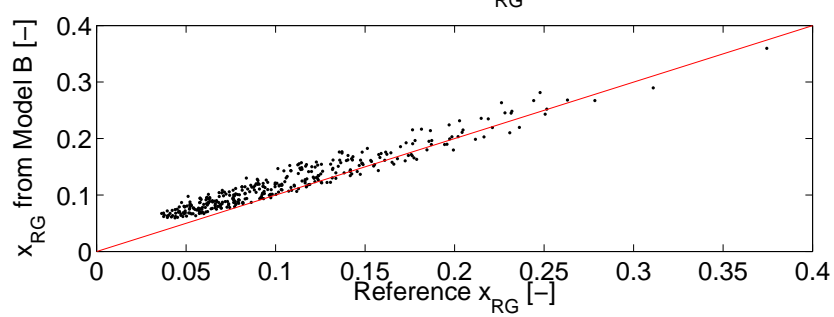

(a)

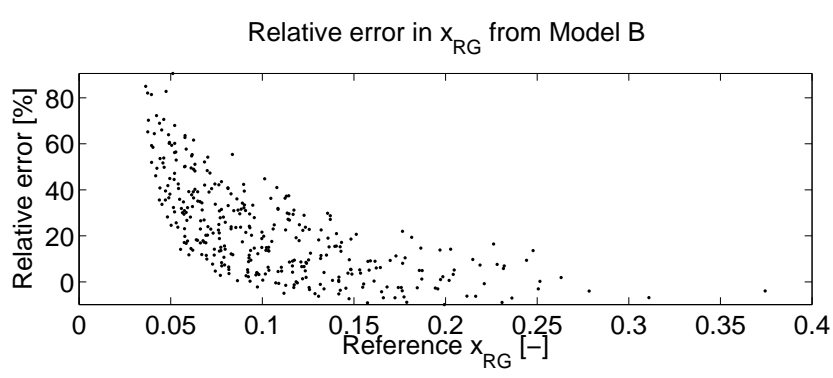

(b)

Figure 4: There is an offset and a scale error in the residual mass fraction from Model B (a). Studying the relative error for Model B shows that the relative error is as large as $80 \%$ for small residual fractions (b).

The method relies on Equation (10). The equation is repeated here for simplicity

$$
x_{R G}=\frac{c_{v, F C}\left(T_{I V C}-T_{F C}\right)}{c_{v, R G}\left(T_{R G}-T_{I V C}\right)+c_{v, F C}\left(T_{I V C}-T_{F C}\right)}
$$

Given the correct temperatures obtained from the reference model, the mass fractions obtained also agrees well with the values from the reference model. It is therefore concluded that the error lies in the temperature estimates. Studying Equation (5)-(9) that leads up to Equation (10), it can be noted that

1. $T_{I V C}$ is sensitive to errors in $p_{c y l}$ and it is therefore important that the method of adjusting the measured $p_{c y l}$ is chosen well. It is especially important that the intake valve is really closed for the offset estimation to work which poses interesting problems with a VCT engine.

2. $T_{F C}$ is subject to charge heating from the cylinder walls which has shown to introduce an error up to about $10 \%$ in the estimation of $T_{F C}$. This is not included in Equation (5)-(9). Another issue is that charge cooling is not included in the reference model.

3. $T_{R G}$ is obtained from a model that is not intended for use with a VCT engine. See for example the result from the parameter tuning in the $T_{R G}$ model to the residual temperature of the reference model in Figure 15, Appendix C. 


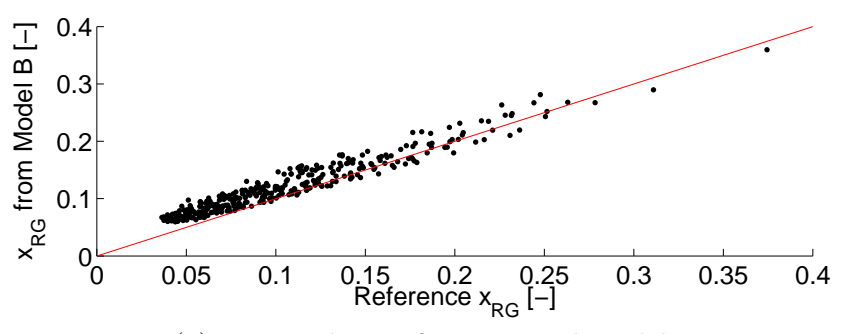

(a) $T_{R G}$ and $T_{F C}$ from original model

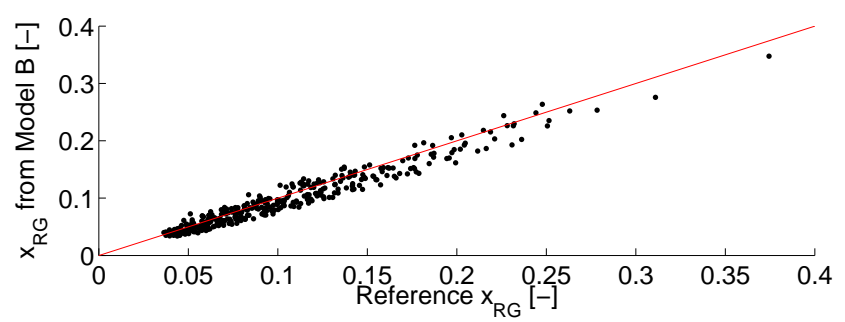

(b) $T_{R G}$ from original model and $10 \%$ increase in $T_{F C}$

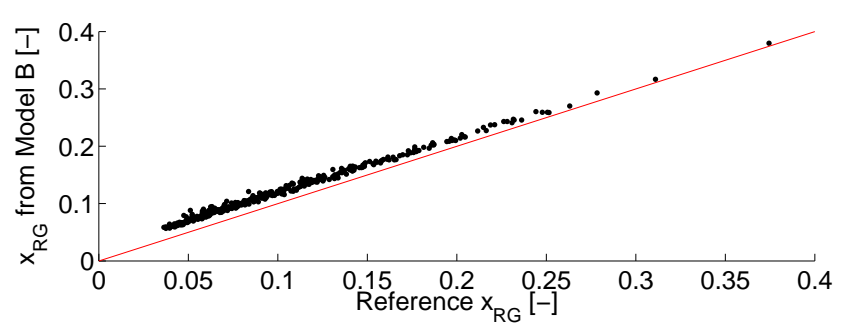

(c) $T_{R G}$ from reference model and $T_{F C}$ from original model

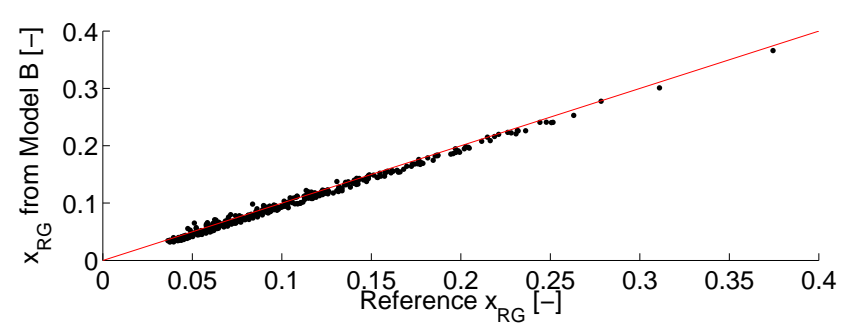

(d) $T_{R G}$ from reference model and $10 \%$ increase in $T_{F C}$

Figure 5: Using the original model for $T_{R G}$ and $T_{F C}$ gives a slight offset for small residual fractions (a). Adding a $10 \%$ heat effect on $T_{F C}$ completely removes this offset (b). Using $T_{R G}$ from the reference model and $T_{F C}$ from the original model makes the spreadage smaller but does not affect the offset (c). Using the correct $T_{R G}$ and a $10 \%$ adjustment to $T_{F C}$ however makes the method perform very well. Note that the $10 \%$ heat correction is an ad hoc value and hence may significantly contribute to the remaining errors $(d)$.
However, the $T_{I V C}$ estimate will be correct since the method is used on simulated data. This is because there is no offset in $p_{c y l}$ and that all other parameters in Equation (7) are well known.

In Figure 5 corrected $T_{R G}$ and $T_{F C}$ estimates have been used to illustrate the methods sensitivity to errors in these estimates. The correction for $T_{R G}$ consisted of replacing the modeled value with the correct value from the reference model. For $T_{F C}$ a $10 \%$ increase to compensate for the charge heating were added.

Looking at Figure 5(b) it is possible to see that a $10 \%$ addition to $T_{F C}$ to compensate for charge heating solves the immediate problems for the method. Figure 5(c) shows that using the correct $T_{R G}$ has a much smaller effect on the result even though the relative error in $T_{R G}$ is in the same range. The error in $5(\mathrm{~d})$, where both compensations have been used, is even smaller. The important observation is however that the method is more sensitive to errors in the $T_{F C}$ estimates than errors in $T_{R G}$ estimates.

Note also that the remaining error in $5(\mathrm{~d})$ is not only due to model imperfections but also from the fact that the $10 \%$ heat addition is not necessarily a good model of the heat exchange. The heat addition may very well vary between operating points.

In Figure 6 the relative error for the variant with $10 \%$ correction of $T_{F C}$ but with the original $T_{R G}$ from the model, is shown. This represents a relative error that is obtainable if the charge heating is included in the model. Note that the errors are well under $30 \%$ except for one outlier and that this is half the error of Model A.

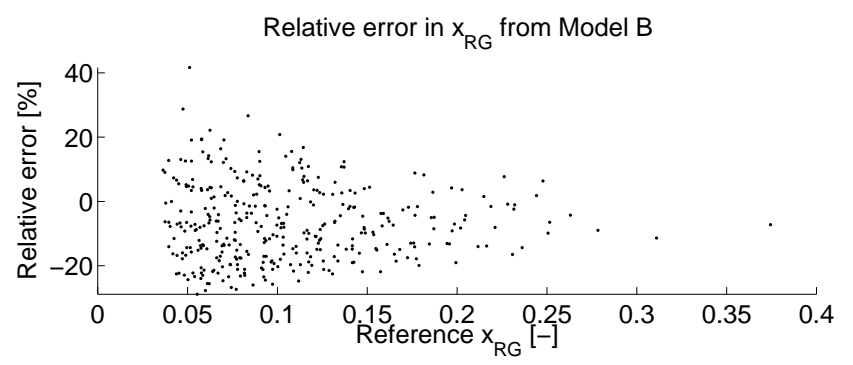

Figure 6: Looking at the relative error it can be concluded that using a $10 \%$ heat compensation in $T_{F C}$ takes care of the larger errors and takes the relative error down to a maximum about $30 \%$ 


\section{Model C}

Model $\mathrm{C}$ is the only model that gives both residual mass fraction and total mass in the cylinder. Model $\mathrm{C}$ suffers from the same problems with $T_{F C}$ underestimating as Model B since they share Equation (8) and (10) with each other. A $10 \%$ increase in $T_{F C}$ is therefore added from the start.

As can be seen in Figure 7 Model $\mathrm{C}$ is quite accurate except for a small offset in the mid range air flows.

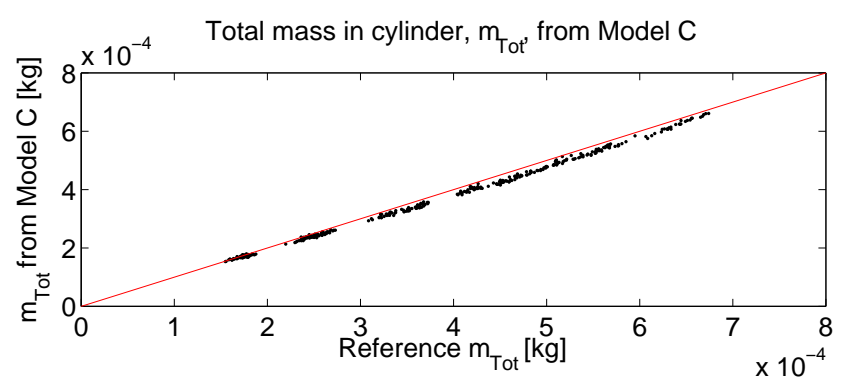

(a)

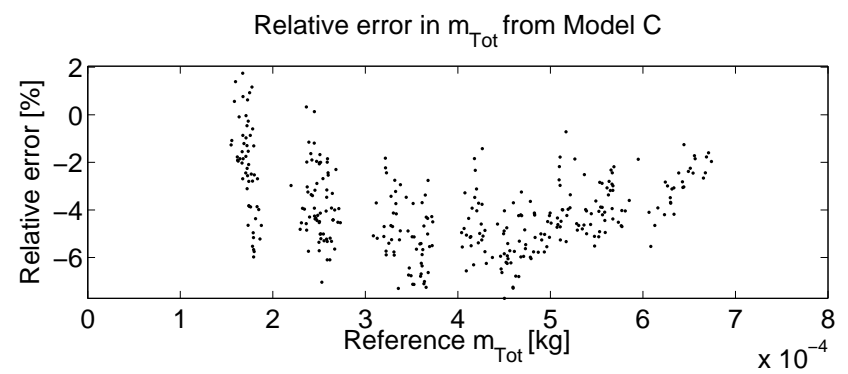

(b)

Figure 7: Total mass estimation using the $T_{F C}$ increase works rather well for Model C. There is however a small error for medium mass flows in (a). The relative error in (b) also shows this dependence on airflow.

When studying the residual mass fraction instead, the model behaves well as can be seen in Figure 8. The spread in residual fraction is however larger than for Model $\mathrm{B}$. The only difference between Model B and Model C is that Model $\mathrm{C}$ relies on its own mass estimate for the estimation of $x_{R G}$. A small error of about $6 \%$ in the mass charge is thus, for some operating points, propagated through the model to the estimate of $x_{R G}$ where it causes an error of up till $50 \%$ extra relative error.

As with Model B the model has been used with the correct values for correlated quantities, i.e. correct values of $T_{R G}$ and $q_{\text {Cooling. }}$. The results are shown in Figure 9 and 10. The results are somewhat better than when using only the charge heating effect but the errors in the mass estimate still affect the residual estimate. Note that the $10 \%$ heat addition only is an ad hoc correction to the $T_{F C}$ estimates and thus that the remaining errors in $T_{F C}$ may be a major contributor to the remaining errors in the $x_{R G}$ and $m_{T o t}$ estimates.

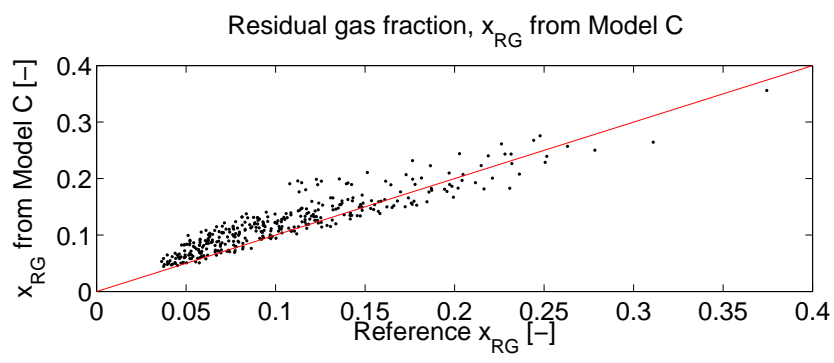

(a)

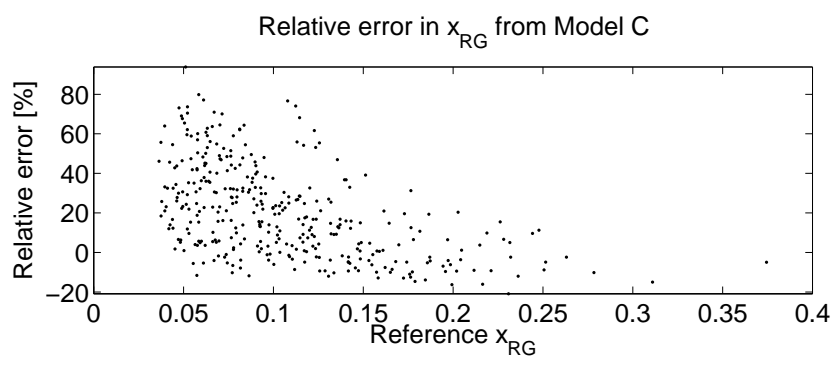

(b)

Figure 8: The spread in the residual mass estimation using the $10 \%$ increase of $T_{F C}$ is larger than for Model B (a). The maximal relative error in (b) is about $75 \%$.

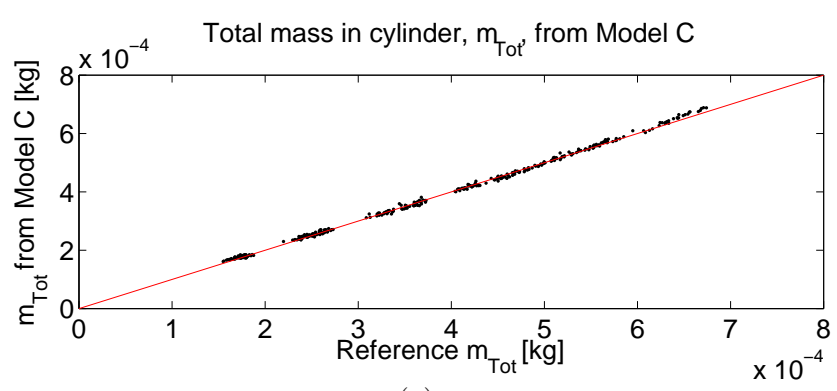

(a)

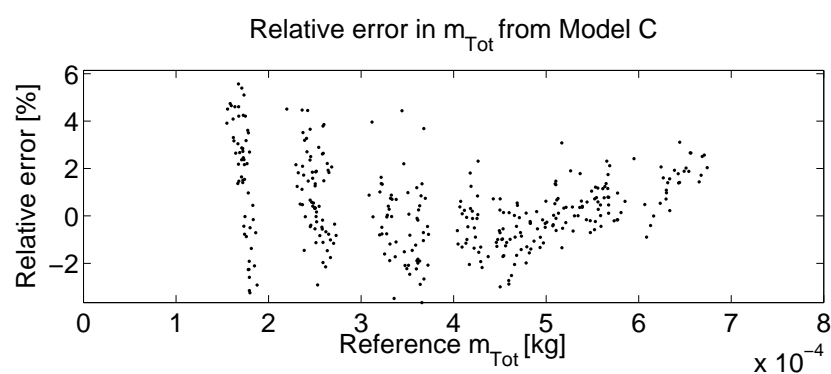

(b)

Figure 9: Using the correct values for $q_{\text {Cooling }}$ and $T_{R G}$ as well as the $10 \%$ increase of $T_{F C}$ eliminates much of the error in the total mass estimate in (a). This also shows up in the relative error in (b) which is now centered about zero. 


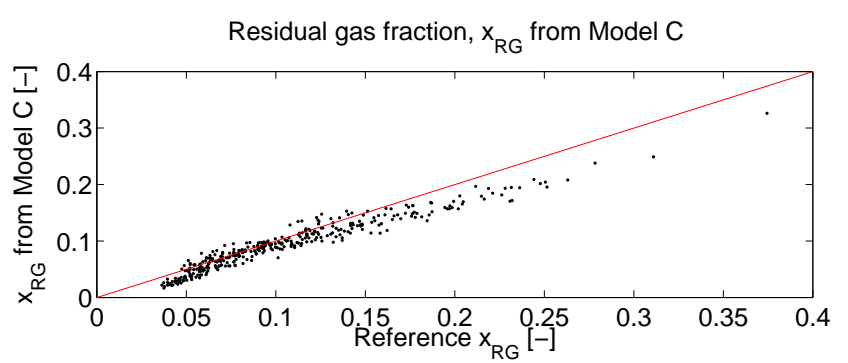

(a)

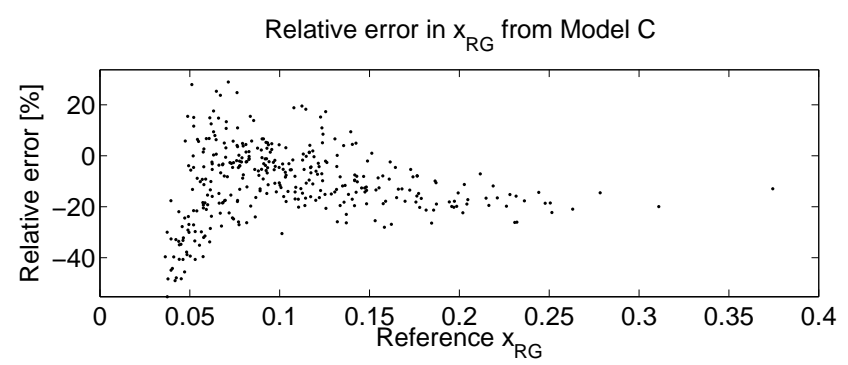

(b)

Figure 10: Using the correct values for $q_{\text {Cooling }}$ and $T_{R G}$ together with the $10 \%$ increase of $T_{F C}$ still leaves a quite large error in $x_{R G}(\mathrm{a})$. However, looking at the relative error in (b) the maximal error is down to about $50 \%$. Note that $10 \%$ is just an ad hoc value.

\section{CONCLUSIONS}

Three models, that predict residual mass fraction which of one also predicts air charge, have been investigated.

Model A gives good results right away considered that is very simple. There are however effects that are not taken care of and the relative error for small residual mass fractions is as large as $70 \%$.

Model B performs well on simulations even though it is sensitive to the mean gas temperature at IVC, $T_{I V C}$, and the temperature of the fresh gas at IVC, $T_{F C}$. The error in temperature for the residual gas, $T_{R G}$, that is introduced by using the $T_{R G}$ model in Equation (9), does not however significantly effect the $x_{R G}$ estimate.

Because of the sensitivity to $T_{I V C}$ and $T_{F C}$ proper care have to be taken when applying the model to measured data since for measured data large errors may be introduced. Pressure measurements, air mass measurements, charge heating effects and fuel evaporation effects are especially important.

Model $\mathrm{C}$ estimates the air mass charge well. However the small errors in mass propagate to the estimates of the residual mass fraction that are affected in a negative manner. This effect is coupled to the sensitivity to $T_{I V C}$ and $T_{F C}$ in Model B.

The sensitivity to $T_{I V C}$ and $T_{F C}$ in Model B and Model C introduces larger errors in the residual mass estimate than the errors introduced by the $q_{\text {Cooling }}$ and $T_{R G}$ models. The issues with $T_{I V C}$ and $T_{F C}$ is the same for both VCT and conventional engines even though the models for $q_{\text {Cooling }}$ and $T_{R G}$ works better for a conventional engine.

Proper handling of Model B and Model C is crucial for good performance while Model A gives good results right away.

\section{REFERENCES}

[1] Jonathan W. Fox, Wai K. Cheng, and John B. Heywood. A model for predicting residual gas fraction in spark-ignition engines. SAE Technical Paper 931025, 1993.

[2] F. Ponti, J. C. Pianai, and R Suglia. Residual gas model for on-line estimation for inlet and exhaust continous vvt engine configuration. IFAC World Congress, 2004.

[3] Michael Mladek and Christopher H. Onder. A model for the estimation of inducted air mass and the residual gas fraction using cylinder pressure measurements. SAE Technical Paper 2000-01-0958, 2000.

[4] Mrdjan Jankovic and Stephen W. Magner. Variable cam timing: Consequences to automotive engine control design. IFAC World Congress, 2002.

[5] Steve Magner, Mrdjan Jankovic, and Stephen Cooper. Methods to reduce air-charge characterization data for high degree of freedom engines. SAE Technical Paper 2004-01-0903, 2004.

[6] Ali Umut Genc. Linear Parameter-Varying Modelling and Robust Control of Variable Cam Timing Engines. $\mathrm{PhD}$ thesis, Wolfson College Cambridge, 2002.

[7] Ylva Nilsson and Lars Eriksson. A new formulation of multi-zone combustion engine models. IFAC Workshop: Advances in Automotive Control, pages 629634, Karlsruhe, Germany, 2001.

[8] G. Woschni. A universally applicable equation for the instantaneous heat transfer coefficient in the internal combustion engine. SAE Technical Paper 670931, 1967.

[9] I.I. Vibe. Brennverlauf und Kreisprocess von Verbennungsmotoren. VEB Verlag Technik Berlin, 1970. German translation of the russian original.

[10] Lars Eriksson. CHEPP - A chemical equilibrium program package for matlab. In Modeling of Spark Ignition Engines, number 2004-01-1460 in SAE Technical paper series SP-1830, 2004.

[11] Gerald M. Rassweiler and Lloyd Withrow. Motion pictures of engine flames correlated with pressure cards. SAE Technical Paper 380139, 1938. 


\section{A NOMENCLATURE}

\section{Abbreviations}

EGR Exhaust Gas Recirculation

VVT Variable Valve Timing

VCT Variable Cam Timing

IVC Intake Valve Closure

TDC Top Ded Center

EVC Exhaust Valve Closure

\section{Used indices}

IVC At Intake valve closure

$C S \quad$ At combustion start

$50 \quad$ At $x_{b}=50 \%$

$B E \quad$ At end of combustion

comp During compression

exp During expansion

$R G \quad$ For Residual Gas part

FC For Fresh Charge part

Tot Total

im In Intake Manifold

em In Exhaust Manifold

\section{Special quantities}

$p_{s, i} \quad$ Sample $i$ of simulated cylinder pressure

$p_{m, i} \quad$ Sample $i$ of measured cylinder pressure

$p_{o} \quad$ Cylinder pressure sensor offset

$m_{\text {air }, s} \quad$ Simulated air mass per combustion

$m_{\text {air }, m} \quad$ Measured air mass per combustion

$\bar{T} \quad$ Average temperature

$A F_{s} \quad$ Stoichiometric air fuel ratio

$\lambda \quad$ Normalized air fuel ratio

$\Phi \quad$ Normalized fuel air ratio

$q_{\text {Cooling }}$ Heat loss due to cooling

$q_{H V} \quad$ Higher heating value of fuel

$x_{c} \quad$ Maximal combustion due to imperfections

$p_{c y l} \quad$ Crank angle based cylinder pressure

$c_{v} \quad$ Specific heating value for constant volume

$c_{p} \quad$ Specific heating value for constant pressure

$r_{c} \quad$ Compression ratio

$\gamma \quad$ Ratio of specific heating values $\left(\frac{c_{p}}{c_{v}}\right)$

OF Overlap factor

$V_{d} \quad$ Displacement volume of engine

$N \quad$ Engine speed in rps

$D_{i} \quad$ Inner seat diameter of intake valve

$D_{e} \quad$ Inner seat diameter of exhaust valve

$A_{e f f, i} \quad$ Effective flow area of intake valve

$A_{\text {eff,e }} \quad$ Effective flow are of exhaust valve

$L_{i} \quad$ Intake valve lift

$L_{e} \quad$ Exhaust valve lift

$x_{R G} \quad$ Residual mass fraction

$x_{b} \quad$ Burn ratio

\begin{tabular}{|c|c|c|}
\hline Parameter & Value & Description \\
\hline$\phi_{i m}$ & $1[-]$ & $\begin{array}{l}\text { Intake manifold fuel air } \\
\text { ratio }\end{array}$ \\
\hline$C_{d, e v}$ & $0.8[-]$ & $\begin{array}{l}\text { Intake valve discharge } \\
\text { coefficient }\end{array}$ \\
\hline$C_{d, i v}$ & $0.8[-]$ & $\begin{array}{l}\text { Exhaust valve discharge } \\
\text { coefficient }\end{array}$ \\
\hline$T_{\text {wall }}$ & $470[\mathrm{~K}]$ & Cylinder wall temperature \\
\hline$p_{i m}$ & (measured) & $\begin{array}{l}\text { Mean value of intake } \\
\text { manifold pressure }\end{array}$ \\
\hline$T_{i m}$ & (measured) & $\begin{array}{l}\text { Mean value of intake } \\
\text { manifold temperature }\end{array}$ \\
\hline$p_{\text {em }}$ & (measured) & $\begin{array}{l}\text { Mean value of exhaust } \\
\text { manifold pressure }\end{array}$ \\
\hline$T_{e m}$ & (measured) & $\begin{array}{l}\text { Mean value of exhaust } \\
\text { manifold temperature }\end{array}$ \\
\hline$C_{1, \text { heat }}$ & 1 & $\begin{array}{l}\text { Woschni heat transfer } \\
\text { coefficient }\end{array}$ \\
\hline$C_{2, \text { heat }}$ & 0.4386 & $\begin{array}{l}\text { Woschni heat transfer } \\
\text { coefficient }\end{array}$ \\
\hline$\theta_{s, v i b e}$ & (estimated) & Vibe start of combustion \\
\hline$\theta_{e, v i b e}$ & (estimated) & Vibe end of combustion \\
\hline$m_{\text {vibe }}$ & (estimated) & Vibe shaping parameter \\
\hline$a_{v i b e}$ & 6.9 & Vibe shaping parameter \\
\hline
\end{tabular}

Table 1 Parameters and constans for the reference model.

\section{B REFERENCE MODEL}

A list of parameters used when simulating the reference model is shown in Table 1.

The reference model has been fitted to data from measurements in a subsection of $7 \times 7 \times 6 \times 2$ operating points. Altogether 427 of the 588 possible operating points are used because the engine does not produce work for all combinations. The operating point grid consists of 7 exhaust cam phasings, 7 intake cam phasings, 6 airflows and 2 engine speeds. The engine speed is 2000rpm and 4000rpm. The span in air mass and cam position is chosen to cover as much of the engine operating region as possible.

Results from fitting the reference model to these measurements are shown in Figure 11. As can be seen the air charge for the reference model gives a good fit to measurements. The maximal relative error is about $20 \%$. Since there are only two different engine speeds it is possible to see that the dependence on cam timing is large. It has to be stressed that no other parameters than the parameters for the Vibe function are used in the least squares optimization.

For completeness two simulated cylinder pressures are shown together with measurements in Figure 12 and 13. The simulation in Figure 12 represents the best possible fit and the simulation in Figure 13 the worst possible fit. 


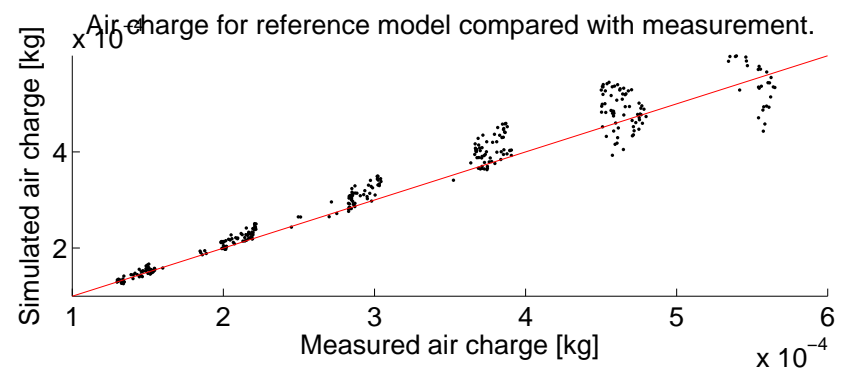

(a)

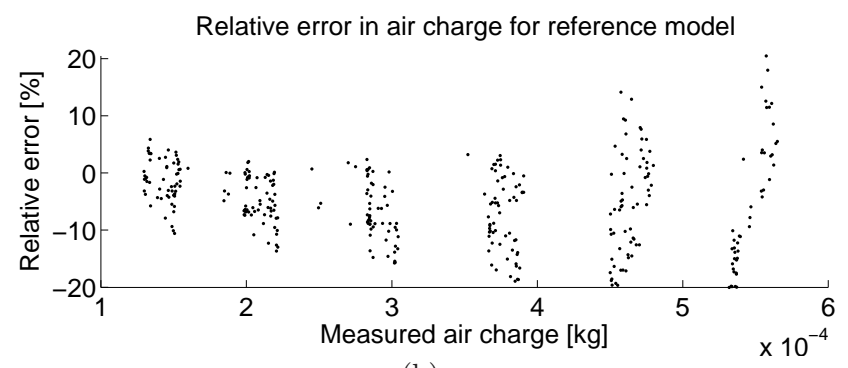

(b)

Figure 11: The air charge for the reference model gives a good fit although with some spreading (a). The maximum relative error is about $20 \%$ (b).

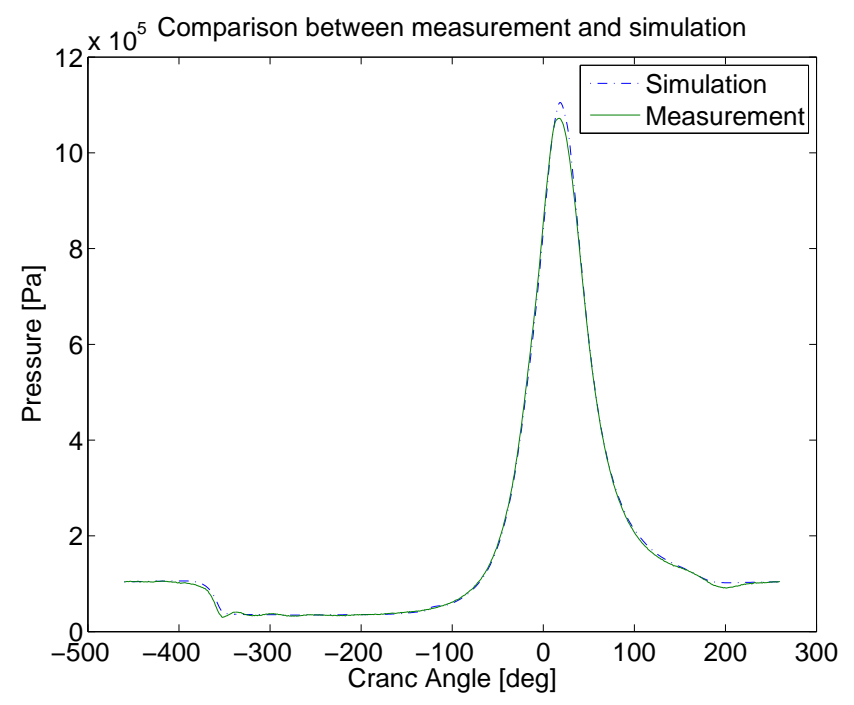

Figure 12: For the best case the accuracy of the reference model is good. The small difference in peak pressure is due to difference between the Vibe function and the actual burn rate. Note that this is the case that gives the smallest mean square error when comparing the measured and simulated cylinder pressure over the whole engine cycle.

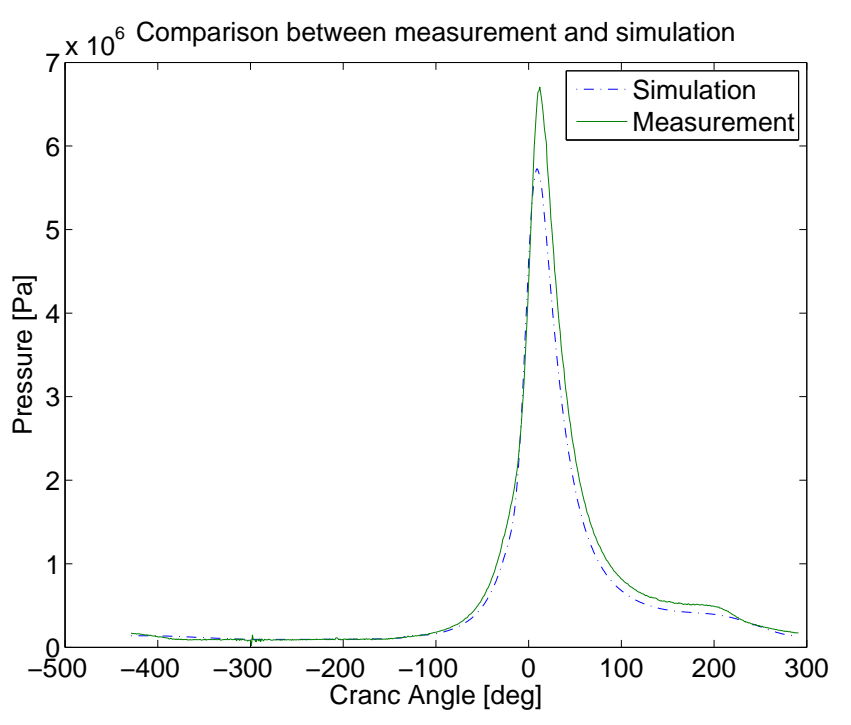

Figure 13: For the worst case the simulated cylinder pressure never reaches the same value as the measured signal. This is due to the fact that the error in air charge is large. 


\section{TUNING OF MODEL PARAMETERS}

Tuning of parameters for Model A The correlation for $x_{R G}$ in Equation (3), Section 4, is directly used in Model A as the single most important component. The equation is repeated here for reference

$$
\begin{aligned}
x_{R G} & =C_{1 x_{R G}} \cdot\left(\frac{p_{e m}}{p_{i m}}\right)^{\left(\frac{\gamma+1}{2 \gamma}\right)} \cdot\left(\frac{O F}{N}\right) \cdot \sqrt{p_{e m}-p_{i m}} \\
& +C_{2 x_{R G}} \cdot \frac{1}{\lambda \cdot r c}\left(\frac{p_{i m}}{p_{e m}}\right)^{\frac{1}{\gamma}}
\end{aligned}
$$

The parameters can be found by using a least square fit or by studying the data in Figure 14. The parameters have been fitted to:

$$
\begin{aligned}
& C_{1 x_{R G}}=0.4205 \\
& C_{2 x_{R G}}=0.5869
\end{aligned}
$$

Model for $\boldsymbol{T}_{\boldsymbol{R}}$ The model for $T_{R G}$ in Equation (9), Section 4, is used both in Model B and Model C. The equation is repeated here for reference

$$
T_{R G}=-\left(C_{1 T_{R G}} \cdot\left(m_{T o t} \cdot N\right)\right)^{C_{2 T_{R G}}}+C_{3 T_{R G}}
$$

Looking at Figure 15 there is little chance of getting a good fit between the data and the model used. This is not surprising since a VCT engine has been used instead of a conventional engine as in [3]. For a VCT engine the residual gas temperature, $T_{R G}$, dependence on cam timing is much stronger than the dependence on mass flow.

The model has been tuned to the data in Figure 15 with the following values

$$
\begin{aligned}
& C_{1 T_{R G}}=0.0033 \\
& C_{2 T_{R G}}=-0.5483 \\
& C_{3 T_{R G}}=1145.5[\mathrm{~K}]
\end{aligned}
$$

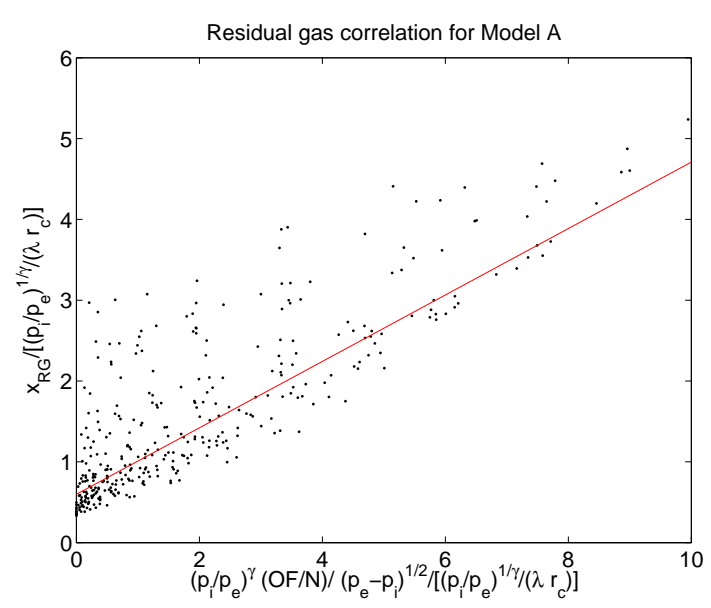

Figure 14: The constants $C_{1 x_{R G}}$ and $C_{2 x_{R G}}$ can be taken directly from the figure or fitted using a least square fit.

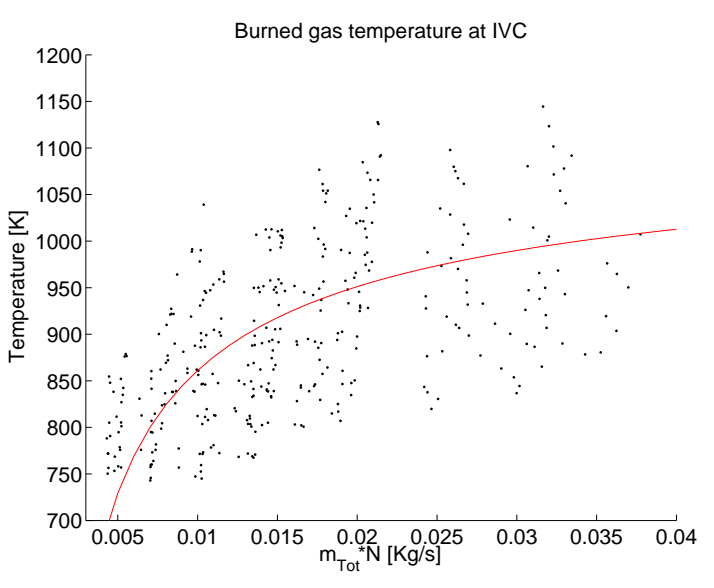

Figure 15: The correlation between mass flow and temperature of residual gas at IVC is weak. The reason for this is that the burned gas temperature varies more in a VCT engine than in an conventional engine.

Model for $\boldsymbol{q}_{\text {Cooling }} q_{\text {Cooling }}$ is introduced in Equation (14), Section 4. The equation is repeated here for reference

$$
q_{\text {Cooling }}=C_{1 q_{\text {cool }}} \cdot \bar{T}_{50}-C_{2 q_{\text {cool }}}
$$

For $q_{\text {Cooling }}$ similar problems occur as with $T_{R G}$. This can be seen in Figure 16. The model has been tuned to the data with the parameters:

$$
\begin{aligned}
& C_{1 q_{\text {Cooling }}}=-9.0622 \times 10^{-6} \\
& C_{2 q_{\text {Cooling }}}=0.0438
\end{aligned}
$$

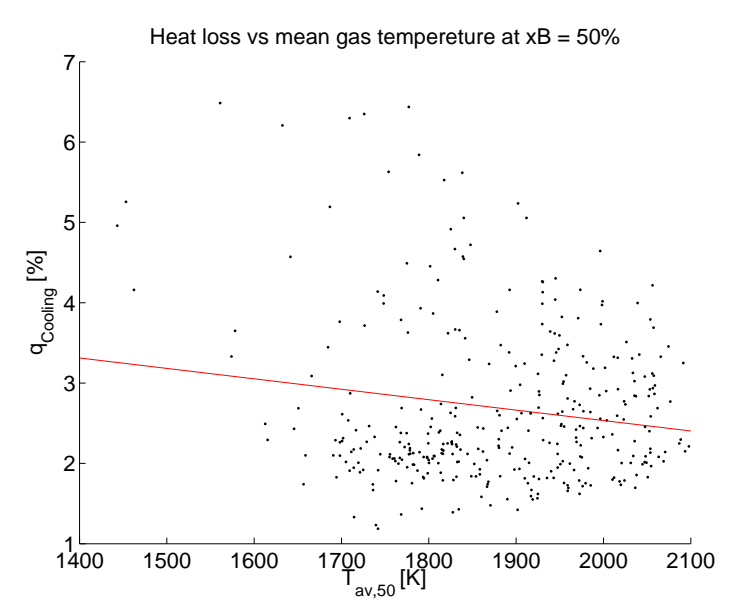

Figure 16: The strong dependence of heat loss on mean gas temperature does not hold for a VCT engine. 